\title{
COMMUNICATION
}

\section{Catalytic Amidation of Unactivated Ester Derivatives Mediated by Trifluoroethanol}

Cite this: DOI: $10.1039 / \times 0 \times x 00000 x$

\author{
Nicola Caldwell, ${ }^{a}$ Craig Jamieson, ${ }^{*}, a$ Iain Simpson ${ }^{b}$ and Allan J. B. Watson ${ }^{a}$
}

Received 00th January 2012,

Accepted 00th January 2012

DOI: $10.1039 / \times 0 \times x 00000 x$

www.rsc.org/

\begin{abstract}
A catalytic amidation method has been developed, employing 2,2,2-trifluoroethanol to facilitate condensation of unactivated esters and amines, enabling the synthesis of a range of amide products in good to excellent yields. Mechanistic studies indicate the reaction proceeds through a trifluoroethanolderived active ester intermediate.
\end{abstract}

Amide bonds are ubiquitous in both natural and synthetic molecules. ${ }^{1}$ This important functionality constitutes the fundamental repeat unit in proteins, which are essential for sustaining biological processes. Amide bonds also commonly feature in small molecules, particularly within a pharmaceutical context. Approximately a quarter of registered drug molecules contain an amide bond ${ }^{2}$ and, consequently, amidation is the most commonly executed transformation in medicinal chemistry laboratories. ${ }^{3,4}$ However, popular methods for the preparation of amides from carboxylic acids utilising coupling agents suffer from inherent drawbacks. ${ }^{5}$ The most significant of these disadvantages from a sustainability perspective is the requirement for stoichiometric quantities of coupling reagent, and the resulting generation of equimolar by-products. Based on this, several catalytic amidation methods have recently emerged which seek to improve the atom economy of this process, thus minimising environmental impact. ${ }^{6-12}$

In relation to the direct conversion of esters to amides, a number of stoichiometric approaches have been developed ${ }^{13,14}$ to overcome the protracted reaction times and high temperatures associated with aminolysis. More latterly, catalytic approaches to aminolysis of ester derivatives have been reported. ${ }^{15-20}$ Despite the obvious utility of these catalytic methods which enable amidation of unactivated ester moieties, there still remain a number of limitations with regard to their practical application. In particular, some of the approaches reported are of limited substrate scope with respect to the acylating species, or rely on the use of rare earth metals which presents a concern from a sustainability perspective.
Within our own laboratories, we have recently focused our attention on the development of catalytic methods for the amidation of simple ester starting materials, with a view to addressing some of the outstanding issues associated with this reaction. We have previously described two mild, efficient processes involving base-catalysed amidation of esters using amino alcohols (Scheme 1). ${ }^{21,22}$ Both of these protocols have been applied to an extensive range of substrates, generating amido-alcohol products exemplified by $\mathbf{1}$ in excellent yields. Mechanistic studies have indicated the progression of the reaction through an ester intermediate (2), followed by rearrangement to the thermodynamically more stable amide. ${ }^{23}$ However, the presence of an alcohol moiety in the starting material in order to facilitate this initial transesterification event was a prerequisite to enable application of this methodology. In order to further develop and extend this catalytic process, we were eager to establish whether exogenous alcohol-derived additives could be employed in this process to form an active ester in situ, thus enabling the direct reaction of simple ester derivatives with amines. Herein, we report our efforts towards the development and exemplification of this catalytic amide bond forming process.

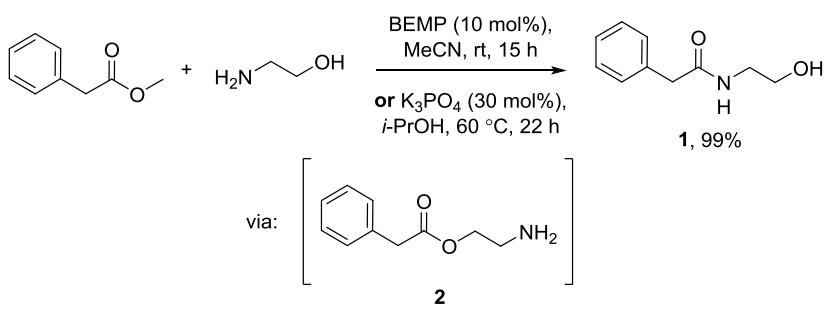

Scheme 1. Base-catalysed amidation of esters.

To assess the feasibility of applying external additives in this methodology, several common peptide-coupling additives were examined in a model reaction examining the conversion of $\mathbf{3}$ to $\mathbf{5}$ (Scheme 2). These additives (HOAt, ${ }^{24} \mathrm{HOBt},{ }^{25} \mathrm{HOCt}^{26}$, Oxyma, ${ }^{27}$ 
$N$-hydroxysuccinamide,

2,2,2-trifluoroethanol,

and hexafluoroisopropanol) were subjected to conditions previously optimised for our progenitor process. ${ }^{21}$ Results of this study indicated that only 2,2,2-trifluoroethanol (TFE) gave any measurable conversion $(6 \%)$ to amide product 5 when used in stoichiometric quantities. No significant conversion was observed for any of the other additives screened, possibly due to the reduced nucleophilicity of these systems, and hence poor reactivity in the initial transesterification step.
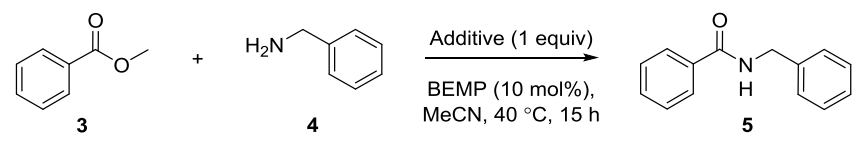

Scheme 1. Screening of additives for direct amidation

Based on this initial survey, TFE was prioritised for further investigation to establish if modifying reaction conditions could improve conversion. To this end, alternative bases (BEMP, DBU, $\mathrm{K}_{3} \mathrm{PO}_{4}, \mathrm{NaH}$, and $t$-BuOK) and solvents (acetonitrile, DMF, NMP, THF, and toluene) were investigated for use in the model reaction. The most effective combinations are summarised in Table $1 .{ }^{28}$ From consideration of this data, both THF and toluene appeared to be effective solvents for this process. Increased conversions to product were observed when using either $\mathrm{K}_{3} \mathrm{PO}_{4}$ or $\mathrm{NaH}$ as the base, however some hydrolysis of the ester starting material was observed in cases where $\mathrm{NaH}$ was employed. Importantly, control reactions with no base or TFE additive consistently gave no conversion as determined by HPLC. As a result of this, and due to the relative ease of handling of $\mathrm{K}_{3} \mathrm{PO}_{4}$, the combination of $\mathrm{K}_{3} \mathrm{PO}_{4}$ in THF was progressed for subsequent optimisation of reaction conditions.

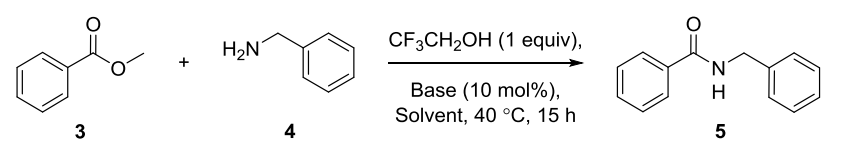

$$
\text { base }
$$
solvent Conversion $(\%)$

$\begin{array}{ccc}\mathrm{K}_{3} \mathrm{PO}_{4} & \text { THF } & 14 \\ \mathrm{NaH} & \text { THF } & 19 \\ \mathrm{~K}_{3} \mathrm{PO}_{4} & \mathrm{PhMe} & 13 \\ \mathrm{NaH} & \mathrm{THF} & 14\end{array}$

Table 1. Screening of alternative bases and solvents. ${ }^{a}$ Determined by HPLC with reference to an internal standard. ${ }^{28}$

In order to expediently optimise reaction conditions using the conversion of ester $\mathbf{3}$ to amide $\mathbf{5}$ as a model process, the powerful statistical approach of Design of Experiments (DoE) was utilised to simultaneously examine the effects of multiple factors in an efficient manner. ${ }^{29}$ A two-level, half-fractional central composite design was selected using Design Expert ${ }^{\mathrm{TM}}$ software. ${ }^{30}$ We envisaged that application of a central composite design in this optimisation campaign would give a more accurate representation of any curvature in the resulting response surface. In this study, we elected to explore the following variables: reaction time $(8-22 \mathrm{~h})$, temperature $\left(40-80^{\circ} \mathrm{C}\right)$, concentration $(0.5-2 \mathrm{M})$, catalyst loading $(0.2-1$ equivalents $)$, and base loading $(0.2-1$ equivalents $){ }^{28}$
From consideration of the raw data, ${ }^{28}$ and inspection of the halfnormal plot associated with the design (Figure 1(a)), it was inferred that perhaps unsurprisingly, temperature was the most significant factor in influencing conversion, with elevated temperatures proving optimal. What was less obvious from the raw data ${ }^{28}$ was the interaction between base and catalyst loading, which appeared to have a significant effect on the conversion (Figure 1(b and c)). An optimum balance appeared to exist between equivalents of TFE and $\mathrm{K}_{3} \mathrm{PO}_{4}$, with conversion rapidly diminishing when catalytic quantities of each were employed. This effect was particularly exacerbated at lower temperatures (Figure $1 \mathrm{~b}$ ) and could be attenuated by performing the reaction at $90{ }^{\circ} \mathrm{C}$. Based on all of the above, it is apparent that using a DoE-based optimisation strategy provides greater levels of process understanding than could be obtained using traditional optimisation approaches, particularly in relation to identifying the interaction between two variables.
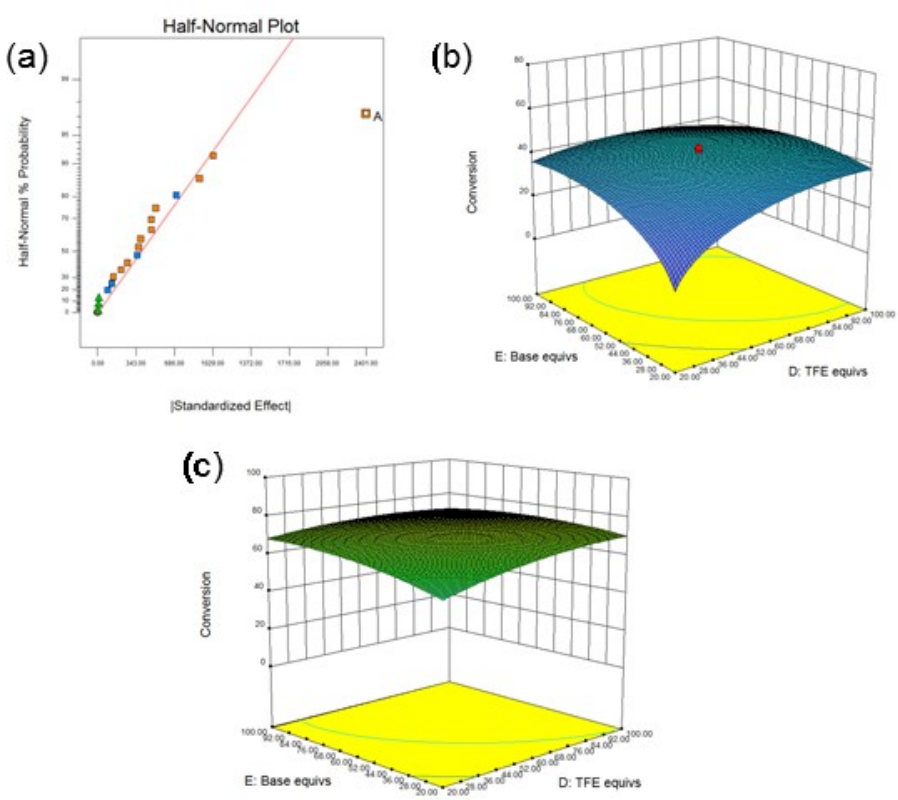

Figure 1. (a) Half-normal plot indicating effect of temperature (A) on conversion; (b) response surface showing effects of TFE and $\mathrm{K}_{3} \mathrm{PO}_{4}$ equivalents on conversion of $\mathbf{3}$ to $\mathbf{5}$, modeled at $60{ }^{\circ} \mathrm{C}$ and (c) response surface showing effects of TFE and $\mathrm{K}_{3} \mathrm{PO}_{4}$ equivalents on conversion of $\mathbf{3}$ to $\mathbf{5}$, modeled at $90{ }^{\circ} \mathrm{C}$.

As enabling a catalytic transformation was the primary focus of our design process, we were keen to investigate whether lower catalyst loadings of TFE could be tolerated. Based on analysis of the response surface, we reasoned that using a full equivalent of the relatively inexpensive base could offset the resulting drop in conversion, particularly when the reaction was carried out at higher temperatures (Figure 1(c)). Pleasingly, employing one equivalent of $\mathrm{K}_{3} \mathrm{PO}_{4}$ allowed the reaction to proceed using only $20 \mathrm{~mol} \%$ TFE. These optimised conditions $\left(20 \mathrm{~mol} \% \mathrm{TFE}, 1\right.$ equiv $\mathrm{K}_{3} \mathrm{PO}_{4}, 90{ }^{\circ} \mathrm{C}$, $22 \mathrm{~h})$ resulted in an isolated yield of $86 \%$ for target compound 5 (Scheme 2), representing a significant improvement to the initial conversion of $6 \%$ obtained in the screening phase of the study.

Again, it should be emphasised that carrying out the corresponding control reactions in the absence of either TFE or $\mathrm{K}_{3} \mathrm{PO}_{4}$ resulted in less than $2 \%$ conversion being observed by HPLC (Scheme 2), confirming that the reaction does not proceed via direct aminolysis, and is likely to be facilitated through the formation of a TFE-derived 
ester intermediate. It is interesting to note that the reaction does not proceed in the absence of a potassium derived base, suggesting that any ammonium salt that could result from combination of the amine substrate and TFE is not reactive enough to attack the starting ester, suggesting an important role in the overall reaction mechanism.

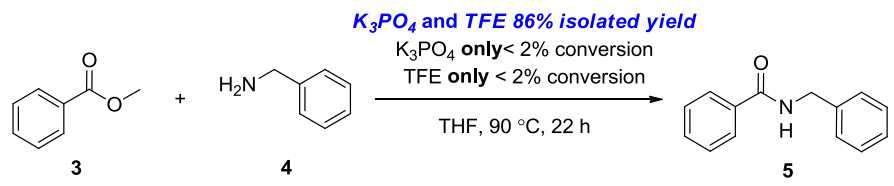

Scheme 2. Optimised conditions in comparison to control reactions.

Having identified optimum reaction conditions using a DoE-based approach, we subsequently sought to investigate the general utility of this methodology. Accordingly, a range of ester and amine starting materials were assessed for their applicability in this reaction manifold (Scheme 2). A broad range of amides could be prepared using the conditions developed in the current study. Several aryl esters were successfully coupled with benzylamine, furnishing good to excellent yields of the corresponding amide products (5-9). The reaction was found to be tolerant of both electron-withdrawing (6-7) and electron-donating (9) functionalities, with the latter exhibiting a slight decrease in yield.

Aliphatic esters were also tolerated as evidenced by examples 10-14, including the use of two amino-acid derived building blocks, (1314). Unfortunately, significant erosion of enantiopurity was observed when using an $\alpha$-chiral ester starting material to afford compound 14 (ee $=8 \%$ as determined by chiral HPLC), representing a potential limitation of this methodology. Efforts are currently ongoing to identify conditions which address this issue.

Esters containing a heterocyclic motif (15-18) were competent substrates; pyridine (15), pyrimidine (16), pyrazine (17), and thiophene-derived (18) esters all gave excellent yields of the corresponding amides. Varying the amine coupling partner facilitated access to a raft of additional amide products. Aliphatic amines such as cyclohexanemethylamine could be successfully employed to form amides with heterocyclic (19) and aliphatic (20) esters. Piperidine (21) and substituted derivatives of piperidine (22) and piperazine (23) represented several examples of effective coupling using a secondary amine. Extending the chain length of the amine starting material to form homologated products such as $\mathbf{2 4}$ and 25 was also tolerated. Finally, we were keen to apply our methodology to the synthesis of a medicinally relevant compound. Accordingly, the experimental Ampakine Farampator (26), which is in phase II clinical trials for ADHD, ${ }^{31}$ could successfully be prepared in one step using our newly developed process.

In the concluding part of our study, additional mechanistic investigations were carried out. As we reasoned that the reaction proceeded via a trifluoroethyl ester intermediate, represented by $\mathbf{2 7}$, this moiety was independently synthesised ${ }^{32}$ and subjected to standard reaction conditions (Figure 2). The conversion of ester derivative, 27, to amide product, 5, was monitored by HPLC over a 22 hour time period. Results from this analysis indicated consumption of the trifluoroethyl ester derivative, with concomitant formation of the desired product. Amide $\mathbf{5}$ was isolated in $77 \%$ yield from this process, which is in broad agreement with previous yield obtained for this substrate. These observations validated our proposal that an active ester derivative was the corresponding acylating species under the optimised reaction conditions. In addition to this, subsequent LCMS analysis of the formation of amide 5 from the methyl ester starting material $\mathbf{3}$ showed trace amounts (4\%) of intermediate 27 throughout the progression of the reaction.

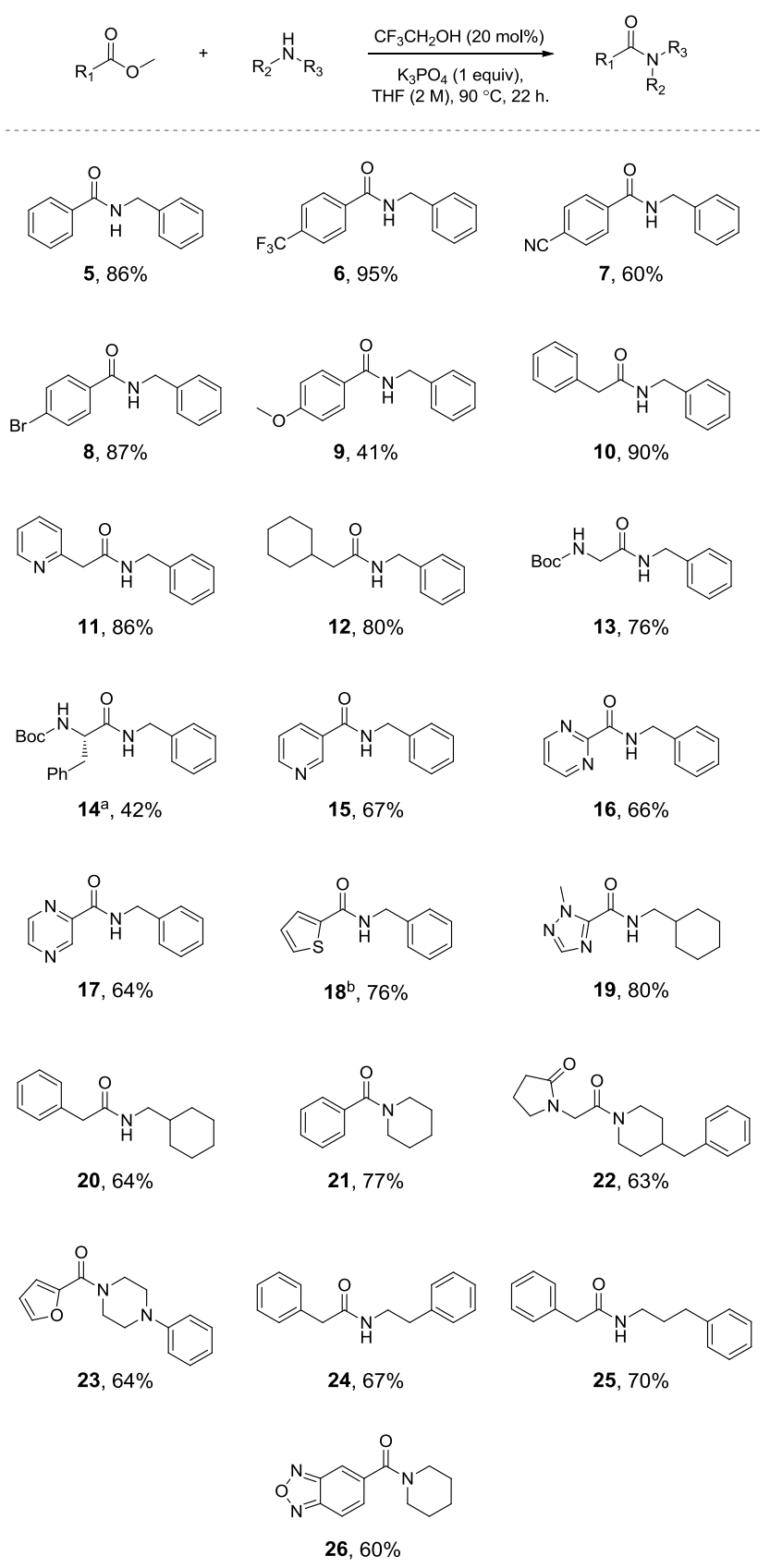

Scheme 2. Assessment of substrate scope. Isolated yields shown. ${ }^{a}$ isolated in $8 \%$ ee as determined by chiral HPLC; ${ }^{28}{ }^{b}$ Synthesised from the corresponding ethyl ester starting material. 

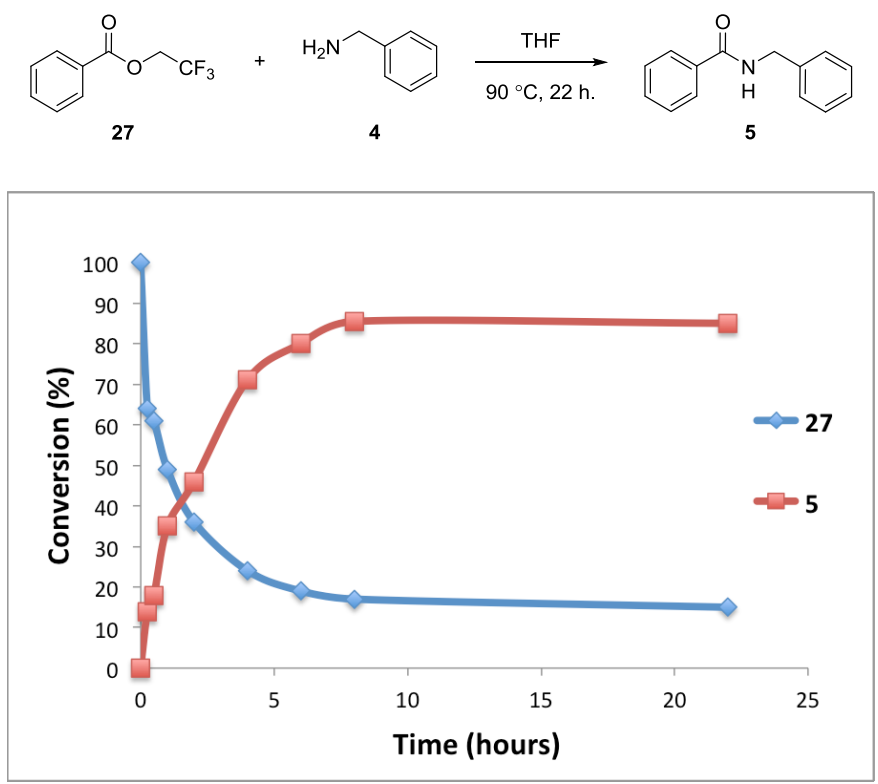

Figure 2. HPLC time study examining the conversion of $\mathbf{2 7}$ to $\mathbf{5}$.

\section{Conclusions}

In summary, we have developed a novel and sustainable organocatalytic amidation method facilitated by 2,2,2trifluoroethanol, an inexpensive and readily available additive. Following initial screening of reaction conditions, expedient optimisation of reaction conditions was achieved using DoE. A wide range of amide derivatives were successfully prepared using this optimised methodology in excellent yields. Subsequent mechanistic investigations confirm progression through a trifluoroethyl ester intermediate and not direct aminolysis. Current work is focused on further expansion of the substrate scope and tuning reaction conditions to enable use of $\alpha$-chiral ester derivatives without epimerisation. The results of these efforts will be reported in due course.

\section{Notes and references}

${ }^{a}$ Department of Pure and Applied Chemistry, University of Strathclyde, 295 Cathedral Street, Glasgow, G1 1XL, UK.

${ }^{b}$ AstraZeneca, Oncology Innovative Medicines, Darwin Building, Unit 310 Cambridge Science Park, Milton Road, Cambridge, CB4 0WG, UK.

Electronic Supplementary Information (ESI) available: experimental procedures and spectroscopic data for all compounds. See DOI: $10.1039 / \mathrm{c} 000000 \mathrm{x} /$

1 N. Sewald and H. D. Jakubke, Peptides: Chemistry and Biology, $2^{\text {nd }}$ edition, Wiley-VCH, 2009;

2 A. K. Ghose, V. N. Viswanadhan and J. J. Wendoloski, J. Comb. Chem., 1999, 1, 55;

3 S. D. Roughley and A. M. Jordan, J. Med. Chem., 2011, 54, 3451;

4 T. W. J. Cooper, I. B. Campbell and S. J. F. Macdonald, Angew. Chem. Int. Ed., 2010, 49, 8082;

5 For a review, see: A. El-Faham and F. Albericio, Chem. Rev., 2011, 111, 6557;

6 For a review, see: R. M. Lanigan and T. D. Sheppard, Eur. J. Org. Chem., 2013, 7453;
7 C. L. Allen, R. Chhatwal and J. M. J. Williams, Chem. Commun., 2012, 48, 666;

8 D. C. Lenstra, F. P. T. J. Rutjes and J. Mecinovic, Chem. Commun. 2014, 50, 5763;

9 C. Gunanathan, Y. Ben-David and D. Milstein, Science, 2007, 317, 790 ;

10 S. Seo and T. J. Marks, Org. Lett., 2008, 10, 317;

11 K. Arnold, A. S. Batsanov, B. Davies and A. Whiting, Green Chem., 2008, 10, 124;

12 N. Gernigon, R. M. Al-Zoubi and D. G. Hall, J. Org. Chem., 2012, 77, 8386;

13 A. Basha, M. Lipton and S. M. Weinreb, Tetrahedron Lett., 1977, 4171;

14 K. Ishihara, Y. Kuroki, N. Hanaki, S. Ohara and H. Yamamoto, J. Am. Chem. Soc., 1996, 118, 1569;

15 T. Ohshima, Y. Hayashi, K. Agura, Y. Fujii, A Yoshiyama, and K. Mashima, Chem. Commun., 2012, 48, 5434;

16 H. Morimoto, R. Fujiwara, Y. Shimizu, K. Morisaki and T. Ohshima, Org. Lett., 2014, 16, 2018;

17 K. E. Price, C. Larrivee-Aboussafy, B. M. Lillie, R. W. McLaughlin, J. Mustakas, K. W. Hettenbach, J. M. Hawkins and R. Vaidyanathan, Org. Lett., 2009, 11, 2003;

18 F. J. Weiberth, Y. Yu, W. Subotkowski and C. Pemberton, Org. Process. Res. Dev., 2012, 16, 1697;

19 X. Yang and V. Birman, Org. Lett., 2009, 11, 1499;

20 C. Han, J. P. Lee, E. Lobkovsky and J. A. Porco, J. Am. Chem. Soc., 2005, 127, 10039;

21 N. Caldwell, C. Jamieson, I. Simpson and T. Tuttle, Org. Lett., 2013, 15, 2506;

22 N. Caldwell, C. Jamieson, I. Simpson and A. J. B. Watson, ACS Sustainable Chem. Eng., 2013, 1, 1339;

23 N. Caldwell, P. S. Campbell, C. Jamieson, F. Potjewyd, I. Simpson and A. J. B. Watson, J. Org. Chem., 2014, 79, 9347;

24 L. A. Carpino, J. Am. Chem. Soc., 1993, 115, 4397;

25 W. Konig and R. Geiger, Chem. Ber., 1970, 103, 788;

26 L. Jiang, A. Davison, G. Tennant and R. Ramage, Tetrahedron, 1998, 54, 14233;

27 R. Subiros-Funosas, R. Prohens, R. Barbas, A. El-Faham and F. Albericio, Chem. Eur. J., 2009, 15, 9394;

28 Full details are reported in the Supporting Information;

29 R. Carlson, Design and Optimisation in Organic Synthesis, Elsevier, Amsterdam, 1992, Chapter 2;

30 Design Expert software is available from Stat-Ease, Inc., Minneapolis, MN, http://statease.com;

31 E. Wezenberg, R. J. Verkes, G. S. F. Ruigt, W. Hulstijn and B. G. C. Sabbe, Neuropsychopharmacol., 2007, 32, 1272;

32 S. Gowrisankar, H. Neumann and M. Beller, Angew. Chem. Int. Ed., $2011, \mathbf{5 0}, 5139$. 